\title{
Staphylococcus aureus and Escherichia coli levels on the hands of theatre staff in three hospitals in Johannesburg, South Africa, before and after handwashing
}

\author{
D O Matuka, ${ }^{1,2}$ MSc Med; B Binta, ${ }^{1}$ MSc Med; H A Carman, ${ }^{1}$ MB BCh, FCDerm (SA); T Singh, ${ }^{1,2} \mathrm{PhD}$ \\ ${ }^{1}$ Immunology and Microbiology, National Institute for Occupational Health, National Health Laboratory Service, Johannesburg, \\ South Africa \\ ${ }^{2}$ Department of Clinical Microbiology and Infectious Disease, School of Pathology, Faculty of Health Sciences, University of \\ the Witwatersrand, Johannesburg, South Africa
}

Corresponding author: D O Matuka (onnicah.matuka@nioh.nhls.ac.za)

Background. Hand hygiene is a fundamental component of infection control. Hand contamination with Staphylococcus aureus and Escherichia coli may contribute to infections.

Objectives. To assess the effectiveness of different handwashing methods in reducing the levels of bacterial flora, especially $S$. aureus and E. coli, on the hands of theatre staff.

Methods. A cross-sectional study was conducted among 70 staff in surgical theatres of three randomly chosen hospitals in Johannesburg, South Africa. Samples were taken before and after handwashing using the modified glove juice method and the fingernail press technique. Standard microbiological techniques were used to identify bacteria. Descriptive statistics and non-parametric analysis were used to compare the differences between hospitals and to determine the effects of handwashing on microbial flora and skin irritation.

Results. S. aureus organisms were isolated in the prewash samples of $29(41 \%)$ and in the postwash samples of 20 (29\%) workers. Of the 29 with positive prewash cultures, 19 (65.5\%) showed decreased postwash counts, while 10 (34.5\%) showed no change or increased counts. Four workers with a negative prewash count had a positive postwash count. No statistical differences were found between postwash counts categorised by the type of cleansing formula used and the washing technique. E. coli organisms were identified in the prewash count of the fingertip press of one worker.

Conclusions. Almost half of the theatre staff carried S. aureus isolates on their hands prior to handwashing and approximately one-third after handwashing. Closer monitoring of handwashing techniques should be introduced.

S Afr Med J 2018;108(6):474-476. DOI:10.7196/SAMJ.2018.v108i6.12485

Hospital theatres are considered to be one of the most complex and hazardous environments that pose a high risk of infection to medical staff (and patients) owing to long exposures to biological agents. ${ }^{[1]}$ Hospital-acquired infections (HAIs) are a serious health issue and an economic burden worldwide, as they present a risk to healthcare workers (HCWs), patients and the community. The main sources of HAIs are contaminated air, contact surfaces and hands of medical staff. HCWs can also acquire these pathogens during direct contact with patients or contaminated environmental surfaces. ${ }^{[2,3]} \mathrm{In}$ spite of hand hygiene protocols and policies in healthcare facilities, poor handwashing compliance continues to exist among medical professionals. ${ }^{[4]}$

Skin flora contains resident bacteria that inhabit the deeper skin layers and are difficult to eradicate (e.g. Micrococcus spp.), as well as transient flora that colonise the superficial layer of the skin and are responsible for nosocomial infections, although they are easily removed by handwashing. ${ }^{[5]}$ Numerous reports reflect the necessity for handwashing using various techniques to prevent the spread of pathogenic organisms to patients. ${ }^{[6]}$ Staphylococcus aureus organisms cultured from healthy hands of medical professionals (10\%) and from damaged hands (16.7\%) were also noted. ${ }^{[3]}$ Studies reported high levels of S.aureus on the hands of female and male HCWs, ranging from $5 \%$ to $20 \% .^{[7-9]}$

S. aureus is a dangerous pathogen that can cause serious and life-threatening diseases, such as severe septicaemia, pneumonia, meningitis, septic arthritis, folliculitis, impetigo, osteomyelitis and toxic shock syndrome. ${ }^{[4,10]}$ Furthermore, methicillin-resistant S. aureus has become common in hospitals and communities. ${ }^{[1]}$ From January to July 2012, there were 1148 South African (SA) cases of confirmed S. aureus bacteraemia. ${ }^{[12]}$ Of these, 619 (54\%) were reported in Gauteng, and 289 (44\%) were resistant to oxacillin/ methicillin, with compliance of only $40 \%$ in intensive care units.

The objective of this study was to assess the efficacy of different handwashing techniques in reducing the levels of bacterial flora, especially S. aureus and Escherichia coli, on the hands of theatre workers.

\section{Methods}

A cross-sectional study was conducted from October to December 2013 and in July 2014 among 70 surgical theatres staff of three hospitals in Johannesburg, SA. These hospitals were selected randomly by their proximity to the testing laboratory and the willingness of management to participate in the study. To test for bacteria on the skin at the beginning of the work shift, the dominant hand of each participant was inserted into and massaged for 1 minute in a sterile polyethylene bag containing $75 \mathrm{~mL}$ of tryptic soy broth (TSB) with neutralisers $(0.1 \%$ polysorbate $80,0.03 \%$ lecithin and sodium thiosulphate). The procedure was performed before handwashing and repeated immediately after washing and before drying of the hands. The samples were transported on the same day in a cooler 
bag with ice to the National Institute for Occupational Health, Johannesburg, for microbiological analysis. Standard microbiological techniques were employed for bacterial quantification and identification of $S$. aureus and $E$. coli. After vigorous mixing, TSB was diluted (1:10 and $1: 100$ ) and $0.1 \mathrm{~mL}$ was plated onto a tryptic soy agar (TSA) medium, $5 \%$ blood agar with gentamicin and mannitol salt agar (Diagnostic Media Products, SA) for isolation of $S$. aureus, using the aseptic spreading method. The inoculated culture plates were incubated aerobically at $37^{\circ} \mathrm{C}$. Total bacterial count enumeration was performed after 72 hours and reported as colony-forming units (CFU) per mL.

The 5 fingertips of the less dominant hand of participants were pressed with equal pressure onto half plates containing $5 \%$ blood agar and MacConkey agar for 5 seconds to isolate Gram-negative bacteria (no bacterial counts were done). Isolated bacterial colonies from the agar plates were identified by conventional techniques (morphology, haemolysis, lactose fermentation, Gram-staining and microscopy) using a study guide on diagnostic bacteriology. ${ }^{[13]}$ Descriptive statistics and non-parametric analysis using Stata 11 (StataCorp., USA) were employed to compare the differences between handwashing techniques and between hospitals. The level of significance was $p<0.05$.

\section{Results}

Seventy individuals (40 females, 30 males) took part in the study, of whom 32 were nurses, 31 medical doctors and 7 other participants. A total of 280 samples (140 hands and 140 fingerprints) for both pre- and postwashing were collected. Of the 70 participants, 8 had an increase, 58 had a decrease and 4 had no change in total bacterial counts after handwashing. There were no statistically significant differences between the prewash and postwash arithmetic mean counts of $S$. aureus (Table 1$)$ or by hospital $(p \leq 0.10)$.

S. aureus organisms were isolated in the prewash samples of 29/70 (41\%) workers and in 20/70 (29\%) postwash samples. Of the 29 who had positive cultures of S. aureus before washing, 19 (65.5\%) showed a decrease in the postwash count and $10(34.5 \%)$ an increase or no change in bacterial load of the postwash count. The proportion of workers with $S$. aureus in the prewash samples differed between hospitals, even though the difference was not statistically significant (exact test, $p=0.08)$. S. aureus was identified in 12/30 (40\%) workers in hospital A, 5/20 (25\%) in hospital B and 12/20 (60\%) in hospital C. Four workers with a negative prewash count had a positive postwash count.

No statistical differences were found between postwash counts categorised by the type of cleansing formula used and between different techniques (scrub with a brush, scrub without a brush and ordinary handwashing). Chlorhexidine gluconate (Hibiscrub) washing solution was used by $54 / 69$ (78\%) participants, povidone iodine (Betadine) by $13(19 \%)$ and $4 \%$ chlorhexidine gluconate (MediScrub) by $2(3 \%)$. One person (2\%) of the total number of participants $(N=70)$ did not report the use of any soap; $S$. aureus was not isolated from this worker. No difference was seen in workers with and without $S$. aureus for postwash samples based on the type of handwashing agent used (exact test, $p=0.153$ ).

Table 1. Mean Staphylococcus aureus CFUs before and after handwashing

\begin{tabular}{lll}
\hline & Prewash $(\boldsymbol{n}=\mathbf{2 9})$ & Postwash $(\boldsymbol{n}=\mathbf{2 0})$ \\
\hline Arithmetic mean, $\mathrm{CFU} / \mathrm{mL}$ & $1.9 \times 10^{3}$ & $0.5 \times 10^{3}$ \\
Mean $\log _{10}, \mathrm{CFU} / \mathrm{mL}^{*}$ & 3.28 & 2.70 \\
$\begin{array}{l}\text { CFU }=\text { colony-forming units. } \\
\text { } \text { Zero count values are excluded from the mean } \log _{10} \text { calculations. }\end{array}$
\end{tabular}

E. coli organisms were isolated from the prewashed fingertip sample of 1 HCW. Staphylococcus spp. were isolated from fingertips, but could not be identified further for $S$. aureus using half-plates (MacConkey/blood agar).

\section{Discussion}

This is the first SA report to quantify the microbiological burden on the hands of theatre workers and to identify S. aureus and E. coli. The overall minimal bacterial counts for prewashing ranged from 0 to $4.3 \times 10^{5} \mathrm{CFU} / \mathrm{mL}$, whereas counts after handwashing ranged from 0 to $1.4 \times 10^{5} \mathrm{CFU} / \mathrm{mL}$. The mean $\log _{10} \mathrm{CFU} / \mathrm{mL}$ of $S$. aureus was 3.28 and 2.70 pre- and postwashing, respectively.

In contrast to our findings of $74 \%$ total bacterial reduction, in another study a decrease of $95-99 \%$ in bacterial load was demonstrated after performing hand hygiene. ${ }^{[14]}$ The percentage of HCWs with $S$. aureus contamination on their hands is higher in our study than levels previously described. ${ }^{[3,7,15]}$ However, Singh and Singh ${ }^{[14]}$ isolated S. aureus in $70 \%$ of nurses, $60 \%$ of students and $40 \%$ of attendants. Persistent skin colonisation with $S$. aureus was reported ${ }^{[8]}$ and was associated with certain body sites and the environment.

The different hospitals showed no statistical differences in the arithmetic mean counts between prewashing and postwashing counts. This may be attributed to the small sample sizes. The handwashing protocols approved by theatre management did not differ between hospitals.

In 4 cases across the hospitals, the postwash count of $S$. aureus was elevated, although the prewash count was zero. Of the HCWs with $S$. aureus isolates on their hands, 10 had increased counts after handwashing. The elevation in bacterial counts could be owing to the hypothesis that transient microbes are easily removed from the hands by washes of $<30$ seconds, whereas resident bacteria embedded in deeper layers of the skin are not easily removed, regardless of the length of time spent handwashing. ${ }^{[16]}$ The elevated postwash counts may have been due to cross-contamination with contaminated surfaces and/or equipment in theatres and between departments, as proven by researchers. ${ }^{[2-4]}$

In 3 HCWs no bacteria were isolated from their hands for both pre- and postwashing, which could be due to handwashing prior to sampling, even though they had stated that they had not washed their hands - possibly for fear of being excluded. However, in 2 of the 3 workers, Staphylococcus spp. were isolated from their fingerprints before handwashing. This suggests that although their palms or hands were not colonised by bacteria, their fingernails were contaminated.

Transient organisms such as S. aureus and Gram-negative bacteria are acquired by HCWs during contact with patients and contaminated surfaces and could lead to HAIs. ${ }^{[17]}$ Our findings may be indicative of contamination of the soap dispenser lid, the hand lever or the soap residual at the tip of the container, which may have been contaminated by air particles. Studies showed that taps and door knobs are rarely cleaned and therefore contain the highest microbial load. ${ }^{[4]}$ The presence of S. aureus on the hands of a large proportion of theatre staff, even after handwashing, should be reason for concern, as gloves are not puncture proof and there may be bacterial transmission to surgical sites from exposed skin. This is also supported by a study investigating handwashing practices of medical students, which showed an increase in bacterial load on the hands of those who washed with soap after toilet use; this increase was attributed to touching the toilet door knobs. ${ }^{[4]}$

Workers carrying $S$. aureus organisms on their hands may harbour these on other body sites, notably in the nose. Persistence of the organisms found on cultures after handwashing may imply that they are not easily removed from the hands and may be resident. ${ }^{[4]}$ Hand 
cleansing should be performed as per the ' 5 moments of hand hygiene' recommended by the World Health Organization (WHO), equipment should be decontaminated after use, and environmental sites should be regularly and effectively cleaned. ${ }^{[18]}$

Staphylococcus spp. were isolated from both prewash and postwash fingertip samples of the less dominant hand. Although not confirmed, it is possible that $S$. aureus was among the Staphylococcus spp. found on the fingertips. De Alwis et al. ${ }^{[4]}$ found high bacterial loads on the dominant hand after toilet use. Two previous studies demonstrated the presence of $S$. aureus using the fingertip method. ${ }^{[19,20]}$

The current study showed that a higher percentage (23\%) of HCWs who scrubbed their hands with a brush had skin irritation, even though the percentage was not statistically significant. While it is generally accepted that nosocomial infections in patients may be acquired from HCWs, and the importance of hand hygiene is stressed throughout the literature, the former is not always the case. For instance, although a previous study demonstrated postsurgical wound infection with S. aureus in 24 of 214 patients, genetic typing showed that HCWs were not the source of infection. ${ }^{[21]}$ E. coli were isolated from the fingerprints of only $1 \mathrm{HCW}(1 \%)$; this finding is similar to that of Singh and Singh, ${ }^{[14]}$ who also reported $1 \%$ in a study done in India.

A major drawback of the current study is the small sample size. Furthermore, methicillin-resistant $S$. aureus was not identified and should be investigated in a larger population. The study warrants further research into the determinants of poor handwashing outcomes, such as duration of washing, amount of antimicrobial agent used and lather formation, sources of cross-contamination (e.g. taps and detergent containers) and behaviour practices. ${ }^{[21]}$

\section{Conclusion}

S. aureus contamination remains a challenge in healthcare facilities. It was found on the hands of almost half of theatre staff before handwashing for theatre and approximately one-third after handwashing. This may be regarded as medically significant. The type of handwashing technique was not shown to be a key determinant in reducing S. aureus counts; however, it may play a role in skin irritation. Handwashing has been reported as the most effective and inexpensive way to prevent transmission, and more advocacy is needed to ensure that HCWs adhere to strict handwashing and hand-care protocols in view of their fundamental role in infection control. In conclusion, our findings may be used to inform other hospitals that handwashing protocols should be reassessed at regular intervals and further analysed for adequacy. This may help to prevent potential S. aureus outbreaks in healthcare settings. Continuing education on behavioural changes to improve hygiene habits of all staff should be encouraged.

Acknowledgements. The assistance provided by Dr A Mayekisa, O Kgasha, A Fourie and Z Kirsten for fieldwork is gratefully acknowledged. We thank the management of the three hospitals and the participants.
Author contributions. HAC initiated the project and contributed to the manuscript. TS contributed to the conceptualisation of the study and provided intellectual input. BB and DOM analysed samples, interpreted the microbiological information and contributed to the content and revision of the article.

Funding. The study was conducted as part of a research project supported by the National Institute for Occupational Health/National Health Laboratory Service and funded by Galderma and Aspen through the Dermatology Society of South Africa awards.

Conflicts of interest. None.

1. Laham NA. Prevalence of bacterial contamination in general operating theatres in selected hospitals in the Gaza Strip, Palestine. J Infect Publ Health 2012;5(1):43-51. https://doi.org/10.1016/j. jiph.2011.10.006

2. Bardaquim VA, Oliveira-de-Souza CW, de-Melo-Martins D, Soares CA, Paiva de Sousa C. Micobiological characterization of the surface contamination in surgical room areas in a hospital in Sao Paulo (Brazil). Infectio 2014;18(4):130-134. https://doi.org/10.1016/j.infect.2014.05.004

3. Li-sha S, Chun-juan X, Hong-bing J, Wei C, Xiao-feng Z, Xiu-hua L. Spread of Staphylococcus aureus between medical staff and high-frequency contact surfaces in a large metropolitan hospital. Int J Nurs Sci 2015;2(4):366-370. https://doi.org/10.1016/j.ijnss.2015.11.001

4. De Alwis WR, Pakirisamy P, San LW, Xiaofen EC. A study of hand contamination and handwashing De Alwis WR, Pakirisamy P, San LW, Xiaofen EC. A study of hand contamination and handwashing practices among medical students. Int Scholar Res Net 2012:1-5. https://doi.org/10.5402/2012/251483
Otto M. Staphylococcus colonization of the skin and antimicrobial peptides. Expert Rev Dermatol 2010;5(2):183-195.

2010;5(2):183-195.
6. Centers for Disease Control and Prevention. Guideline for hand hygiene in health care settings: Recommendation of the Health Care Infection Control Practices Advisory Committee and the HICPAC/ SHEA/APIC/IDSA in Hand Hygiene Task Force. Morbid Mortal Wkly Rep 2002;51(RR16):1-45.

7. Cespedes C, Miller M, Quagliarello B, Vavagiakis P, Klein RS, Lowry FD. Differences between Staphyloccus aureus isolates from medical and non-medical hospital personnel. J Clin Microbiol 2002;40(7):2594-2597. https://doi.org/10.1128/JCM.40.7.2594-2597.2002

8. Dascher FD. How cost effective is the present use of antiseptics? J Hosp Infect 1988;11(Suppl A):227-235.

9. Tammelin A, Hambraeus A, Stahle E, Ransjo U. Nasal and hand carriage of Staphyloccus aureus in staff at a department for thoracic and cardiovascular surgery: Endogenous or exogenous source. Infect staff at a department for thoracic and cardiovascular surgery: Endogenous or
Control Hosp Epidemiol 2003;24(9):686-689. https://doi.org/10.1086/502277

10. Lowy FI. Staphyloccus aureus infections. N Engl J Med 1998;339(8):520-532. https://doi.org/10.1056/ NEJM199808203390806

11. Chambers HF. The changing epidemiology of Staphylococcus aureus. Emerg Infect Dis 2001;7(2):178182. https://doi.org/10.3201/eid0702.700178

12. National Institute for Communicable Diseases. Germs South Africa Annual Report. 2012. http://www. nicd.ac.za (accessed 4 May 2018).

13. Bartlett MA. Diagnostic Bacteriology: A Study Guide. Philadelphia, USA: FA Davis, 2000:53-61.

14. Singh S, Singh AK. Prevalence of bacteria contaminating the hands of healthcare workers during routine patient care: A hospital-based study. J Acad Clin Microbiol 2016;18(1):60-62. https://doi. routine patient care: A hospita
org $/ 10.4103 / 0972-1282.184764$

15. Rocha LA, Ferreira de Almeida E, Borges L, Gontijo Filho PP. Changes in hands microbiota associated with skin damage because of hand hygiene procedures on the health care workers. Am J Infect Control 2009;37(2):155-159. https://doi.org/10.1006/j.ajic.2008.04.251

16. Jensen D, Macinga D, Shumaker D, Bellino R, Arogast J, Schaffner D. Quantifying the effects of wate temperature, soap volume, lather time and antimicrobial soap as variables in the removal of Escherichic coli ATCC 11229 from hands. J Food Protect 2017;80(6):1022-1031. https://doi.org/10.4315/0362 028X.JFP-16-370

17. Nicolay CR. Hand hygiene: An evidence-based review for surgeons. Int J Surg 2006;4(1):53-65. https:// doi.org/10.1016/j.jhin.2005.06.002

18. Curran ET. Outbreak column 14: Staphylococcus aureus - new outbreaks of old infections. J Infect Purran ET. Outbreak column 14: Staphylococcus aureus - new outbreaks of
Prevent 2014;15(4):148-153. https://doi.org/10.1177//1757177414536942

19. Creamer E, Dorrian S, Dolan A, et al. When are the hands of health care workers positive for methicillin-resistant Staphylococcus aureus? J Hosp Infect 2010;75(2):107-111. https://doi.org/10.1016/ j.jhin.2009.12.005

20. Pittet D, Dharan S, Touveneau S, Sauvan V, Perneger TV. Bacterial contamination of the hands of hospital staff during routine patient care. Arch Intern Med 1999;159(8):821-826. https://doi. org/10.1001/archinte.159.8.821

21. Ahmed AO, van Belkum A, Fahal AH, et al. Nasal carriage of Staphylococcus aureus and epidemiology of surgical site infections in a Sudanese university hospital. J Clin Microbiol 1998;36(12):3614-3618.

Accepted 21 December 2017 